\title{
Do management accounting systems influence organizational change or vice-versa? Evidence from a case of constructive research in the Healthcare Sector
}

\begin{abstract}
The paper aims to analyze the process of change of management accounting system (MAS) as a consequence of changes in the complexity of organizational structure in healthcare. It deepens the factors and elements involved in the process of change of the management accounting systems according with the theoretical frameworks of Habermas (1987) and Laughlin (1991).In this perspective organization structure is seen as a set of tangible and intangible elements, which interact with the external environment. In this respect the organizational changes are seen as the consequence of the interaction between tangible and intangible elements of the organization and between the organization and the external environment.

The process of change was not studied from an external standpoint, but through an active participation and contribution of the researchers in the process of change itself. Using a constructive research approach, the researches were actively involved with the actors of the change in developing the process of change, and in facilitating the overcoming of some cultural gaps and resistance which could arise in professional organization.

The paper provides empirical insights of the characteristics of the process of change of MAS in a Heath Care setting with a particular focus on aspects characterizing the process of change itself. Finding suggest the importance of putting high attention in the development and planning of the process of change itself and underline how the attention to peculiarities of the organization, in to this phase, could make the MAS able to impact on the behaviours and culture of professionals.
\end{abstract}

\section{Introduction}

The aim of this study is to analyze the process of change of management accounting system related to changes in organizational structure, by developing the research in the healthcare (HC) sector.

Healthcare organizations are particularly complex and characterized by a duality: a clinical staff that demands services and an administrative staff that provides support services to the clinical staff (Harris and West 1925; Jacobs et al., 2004). Clinicians and administrative belong to two different dimensions that are loosely coupled (Weick, 1976): they are linked each other but each retains their own identity and they have few elements in common. They represent different blocks in a complex organization.

This situation impacts also on the tools used to measure results and performance of these organizations . Clinicians work on their experience and expertise and their activities are difficult to be measured by formal systems, while accounting measures reflect efficiency objectives derived from the private sector principles, which answer to the requirements of government and that are not aligned with core activities. The role of accounting in this situation may become problematic, with the risk of shifting from informative support for decision making process to a mere formality. The study, in these settings, of the dynamic of change in accounting considering its linkage with organizational transformation is therefore particularly interesting: the pathways of change in $\mathrm{HC}$ organizations find a further element of difficulty in their professional nature and the aforementioned duality. According with the organizational paths of transformation (Laughlin, 1991; Grenwood and Hinings, 1988) a substantial change modify the "genetic code" of organization and finds reflections 
in all the future generations (Smith, 1982): this process in a professional settings needs specific care and support in design and management.

In studying the process of change we were interested not only from an external standpoint. As we will describe in the paper, we were interested in participating and giving a contribution in the process of change, through an internal perspective and a participative approach. Specifically we were interested in describing the characteristics of the process of change of MAS which make the new MAS able to impact on behaviours and culture of individuals, so developing a substantial change. The research questions we are going to answer are: "Does a change in the management accounting system could favour a change in the interpretative scheme, thus bringing to a substantial transformation (morphogenesis)?" and "How the process of change should be developed, considering the role of clinicians?". In doing so, we aim to contribute in providing a framework for understanding and analysing a situation of major transformation in a highly professionalised setting, like HC organizations.

The paper is organized as follows. The first section will provide a literary background. In the second section the theoretical model will be explained and adapted to HC context: in particular we use the Habermasian theory of society in the analysis of changes in organization. The subsequent section will provide a brief description of the Italian healthcare sector and of the specific regional HC system (Tuscany) where this study has been developed: we focus in the organizational change occurred in this region and problems this arose. The fifth section explains the methodology applied to the research setting. In particular we use a constructive approach (Kasanen et al., 1993). Findings will be explained in the sixth session and discussion and conclusion will be developed in the last session.

\section{Background}

The issue of changes in Management Accounting System (MAS) and their relations with the Organizational Structure has been a topic in research agenda for long time. In 1981 Miller (1981) emphasized that a complementary alignment among MAS and Organizational Structure is an important factor in determining performance.

Several studies have analyzed the link between MAS and organizational structure both in the private and in the public sector. Cassia et al. (2005) studied the relationship between organization configuration and MAS in 501 Italian companies through a quantitative analysis. They put in relation a set of attributes of the MAS (i.e. kinds of used techniques, function of accounting etc.) with a set of attributes of the organizational structure (i.e. size, level of delegation, characteristics of the production system etc.). They tried to analyze the process of change, but they didn't observe how changes happened; they observed companies in a specific moment, but in the meanwhile the large sample allowed them to point out the position of companies in a stage of their life cycle: birth, growth and maturity. They found that the greater is the organizational complexity, the higher is the MAS evolution.

Another research by Mooree and Yuen (2001) empirically verified the relationship between MAS and Organization Configuration in the Australian clothing and footwear industry. They analyzed the relationship between evolution of organization configurations and MAS. Firms were divided in five 
clusters representing the life cycle stages (birth, growth, maturity, revival and decline). The assignment to a specific cluster was based on age, sales growth rate and strategic, structural and managerial characteristics. Then attributes of MAS were analyzed. Some example of attributes were: criteria for performance evaluation, mode of aggregation of data, scope of information, timeliness of information etc. They found an internal consistence between stage of the life cycle of the organization and complexity of the MAS, in particular the MAS was found to be more complex and rich of information in the growth stage.

These two studies analyzed, through a contingency framework, the evolution of the MAS in a life cycle perspective. These studies analyzed changes collecting data from several firms and dividing them in clusters correspondent to the stage of the life cycle and put these clusters in relationship with characteristics of the MAS, but they didn't analyze the process of change.

Another study by Jones and Dewing (1997), through a longitudinal analysis in a large acute British hospital, analyzed changes in MAS and its use after the conversion of the organizational structure from a functional structure to a divisional structure based on clinical directors, service managers, operational managers and finance staff. This reform delegated the responsibility as close as possible to the point of delivery (doctors) with the objective to improve performance. The MAS changed in order to represent this different distribution of responsibility. However, this attempt, to make doctors more responsible for costs failed, because the MAS was defined and imposed by the central management in order to exercise a crude control on costs. Moreover doctors were not really involved in the individuation of a system that could have been able to support the system of internal responsibility. Some consequences were a lack of link between financial and non financial information and the production of late and inaccurate reports.

Another study by Chenhall and Langfield-Smith (1998) analyzed the role of the MAS within organizational change programs in three manufacturing firms. In particular they studied the adaptation of the management accounting system and the role of accountants following changes in the organizational structure. An example of organizational change they described was the introduction of work based teams and changes needed in the management accounting system in order to analyze performance. The aim of the introduction of the work based teams was to improve quality, customers' satisfaction and efficiency. The authors of this study found that the development of a performance measurement system was more successful in firms where it was designed through integration between manufactures and management accountants. In these firms such integration allowed the alignment between quality and cost control and improved the credibility and trust of management accountants. This finding suggests the importance of integration of users of the systems and their managers, i.e. the management accountants.

In general, findings of these researches support the importance of considering many issues in analyzing the relationship between MAS and organizational structure and changes in MAS : (1) the process of change as an elements of the change itself (Laughlin, 1991); (2) the importance of the involvement of users of the system in the process of change, especially in professional organization; (3) the need of a collaboration between users of the system and management accountants in the process of change (Cinquini and Campanale, 2010).

An important factor, which needs to be taken into account in the process of change, is the professional bureaucracy which characterises healthcare organizations.

According to Brunsonn (1985), changes in organization and MAS usually require a strong effort in order to overcome problems that arise when an organization is characterized by a strong culture. These problems are of particular interests in healthcare organizations where management and 
decision making process (and performance accordingly) are strongly influenced by professionals that are used to work in complete autonomy.

Empirical research demonstrated that formal control is perceived as the most offensive of autonomy of professionals while it is privileged an environment which emphasizes self control and social control, standardization of skills and group co-ordination mechanism (Abernethy and Stoelwinder, 1990; Abernethy and Vagnoni, 2004).

Moreover Mintzberg (1989) argues that traditional forms of control, such as budget, are ineffective for controlling the work of professionals because they are not able to represent the complexity in the tasks performed.

There is the necessity to build a system which represent the complex tasks performed by professionals (according to Mintzberg argument) and to build it trough the involvement of the same professionals who perform activities; in fact, according to Abernethy and Stoelwinder (1990), professionals accept a system based on standardisation when the system and the supervising originate from their expertise.

\section{Theoretical framework}

The following elements need to be taken into account in studying and understanding organizational and accounting change: (1) how organizational and accounting changes relate and interact each other; (2) what is the nature and the process of organizational change; (3) what is the nature of the organization (Broadbent and Laughlin, 2005).

The third point, the nature of the organization, is of fundamental importance in studying and understanding the process of change. The complexity of organization influences the process of change, the interaction of elements of the organization and the result of the process itself.

Habermas' theory about society (1987), developed and refined by Broadbent and Laughlin (2005), suits our study about the nature of organization and the process of change in healthcare sector.

Habermas traced the society as the combination of three elements: lifeworld, systems and steering media.

Habermas (1987) defined the lifeworld as a cultural space which articulates culture of individuals, society and personality. Culture is the stock of knowledge which individuals use in order to interpret and understand something in the world. Society concerns with some order through which individuals regulate their membership in a social group. Personality concerns with competencies that make a subject capable of speaking and acting and asserting his/her identity. Lifeworld is not static but it evolves according to culture, society and personality.

Systems, such as organizations, are the tangible expressions of the less tangible lifeworld and are guided by the lifeworld itself. Systems emerge when the lifeworld becomes more and more complex and starts to need a tangible expression.

In this context Habermas introduced the third element, the steering media, which are mechanisms such as power, money, law - steering the communication and interaction between lifeworld and systems. Steering media plays a role in assuring that systems reflect lifeworld. But, when systems grow in complexity (due, for example, to capitalism growth) there is the risk that lifeworld and systems become differentiated and decoupled. In this situation there is the possibility that steering media starts to follow the systems and not the lifeworld, thus bringing the systems to influence or colonize the lifeworld. 
Broadbent and Laughlin (2005) refined this model of society and adapted it to an organizational context. They argued that every organization has its own lifeworld, systems and steering media and called these elements respectively interpretative scheme, sub-systems and design archetype, where design archetype balances and makes coherent interpretative schemes and sub-systems. As suggested by Power and Laughlin (1992) accounting, within organizational context, is a steering media.

The coherence between elements of the organization and the external environment is required for the equilibrium of the whole organization (Miller and Friesen, 1984; Mintzberg, 1989).

When the equilibrium between these elements is reached, the organization tends to an inertia and its broad design tends to be stable and resistant to change (Laughlin, 1991; Miller and Friesen, 1984). This inertia could be interrupted only by an environmental disturbance (Laughlin, 1991), which means some external uncontrollable factors that require a change in the organization.

According to Smith (1982) and Robb (1988) two kinds of changes may occur: morphostasis (first order change) and morphogenesis (second order change) ).

Morphostatis occurs when the change in the organization doesn't really affect the core of the organization with a reluctance of the organization to accept the change and a tendency to come back to the pre-existent situation. This change doesn't affect the interpretative scheme of the organization. Morphostatis could act through two different tracks: (1) Rebuttal and (2) Reorientation. Rebuttal occurs when an environmental disturbance is faced with some changes in the design archetype, but after that the disturbance has been rebutted, the design archetype come back to the previous one. Reorientation occurs when the environmental disturbance affects not only the design archetype, but also subsystems. The disturbance can't be rebutted but has to be accepted and internalized into the working of the organization, but the culture (the interpretative scheme) is not affected.

Morphogenesis is a change that penetrates deeply into the core of the organization and brings to permanent modification of the organization. This change affects the interpretative scheme of the organization. Morphogenesis could occur through a (1) colonization or (2) evolution. They both bring to a deep change in the interpretative scheme, but while colonization is a sort of forced change of individuals, evolution is chosen by individuals freely and without compulsion (Laughlin, 1991). Colonization is a more frequent phenomena if compared to evolution.

Many factors could affect the nature of change: morphogenetic or morphostatic. For example Brunsonn (1985) identified ideology as a factor affecting change. Ideologies, as a part of the interpretative scheme, could be weak or strong. Weak ideologies are inconsistent, simple and inclusive, while strong ideologies are consistent, complex and inclusive. Brunsson (1985) argued that organizations with strong ideologies are resistant to fundamental changes in ideologies and therefore to changes in the interpretative scheme. Organizations with weak ideologies are more opened to manipulation and fundamental changes in ideologies and thus in interpretative scheme.

Grenwood and Hinings (1988) analyzed why organizations follow particular tracks in the process of change They found four elements affecting inertia or change: the strength of the constraints and pressures upon change which destabilize the organization; the level of commitment held by participants; the power dependencies; the interests of individuals and the organizational competences and capabilities. Regarding the first factor - constraints - they are related to particular contingencies that modify situational circumstances/contexts which create pressure for a change in the organization. Typical circumstances/contexts considered are environment, technology and size. When this circumstances/contexts change, they create pressure on the organization and produce a 
need for a response. When contingencies create severe ${ }^{1}$ contradictions between circumstances / context and organization a need for a change occurs. Severe contingencies create higher contradictions between the organization and circumstances/context, thus bringing to a greater change. On the other hand, when contingencies do not create contradictions between contingencies/context and organization, inertia is most likely to occur. Regarding the second factor - the level of commitment held by participants - it relates to the attitudes of individuals to promote alternative interpretative schemes or their inertia versus changes. As underlined previously, systems are the expression of interpretative scheme, so the higher is the commitment to the existent interpretative scheme the lower is the possibility for change. Regarding the third factor - the power of dependencies - Grenwood and Hinings (1988) started by arguing that the organizational design serves the interests of some groups of individuals and acts as delineator of advantages and privileges. Organizational arrangements are typically in accordance with a dominant coalition's interpretative scheme. Structural changes are affected by the extent to which the dominant coalition is dissatisfied with the accommodation of its interests, and by the ability of this coalition to protect and express them. In the authors' view, if the dominant coalition is satisfied with its arrangement of interests, organization will tend to inertia, vice-versa, if the dominant coalition is not satisfied with its arrangement of interests, it can operate as a destabilising element. Regarding the fourth factor interests of individuals - they argue that the higher is the level of satisfaction of a group of individuals with a particular arrangement compared with its own interests, the lower is their will to change, and the lower is the level of satisfaction of a group about the present arrangement, the higher is their will to change. Regarding the last factor - the competences and capabilities - the authors underline the role of top managers of the organization in promoting changes. Senior managers are in a position where they are responsible for the evolution of interpretative scheme. As the culture of the senior managers makes him willing and able to change he/she becomes a leader of the change. In order to become leader also skills and knowledge are required. In conclusion, the greater are competences and capabilities (made of culture, skills and knowledge) the greater is the potential for change.

Dunphy and Doug (1988) and Smith (1982) found also that a morphogenetic change is favoured when it occurs through a collaborative approach between individuals in order to facilitate a common organizational vision based on shared values. Laughlin (1991) made a comparison between the European Railways and the Church of England and found the occurrence of a morphogenetic change in the European Railways and of a morphostatic change in the Church of England. Even if the author didn't go deep in the individuation of factors affecting results, he concluded that perhaps church of England could be characterized by a culture similar to the culture of University and Health Authorities. In fact this kind of institution tends to maintain them protected from unnecessary and unwanted intrusion and tend to reject change. A recent research by Cinquini and Campanale (2010) studied the role of the management accounting system in healthcare sector and found that the integration and the collaboration between clinicians and controllers in the development of management accounting systems could allow an higher alignment between subsystems and lifeworld. In this research alignment means mutual adjustment between lifeworld and sub-systems.

\footnotetext{
${ }^{1}$ Severe contingencies mean that there are multiple contingencies that bring to different directions.
} 
Another important factor affecting change is the role of researcher as a supporter in the attainment of a new equilibrium in the elements of the organizations. This is required when a disturbance modifies previous arrangements of the organizations. In fact the researched (employees, doctors, managers etc, who are actors of changes) often need an external support because they could lack of the necessary competencies needed to promote changes. In this respect researcher develops an interaction with researched. This interaction is made of communication and aims at identifying problems to be faced and how to face these problems.

In the Habermasian research, the language is a key element. The language is seen as a discursive process made of actions that researcher has to carry on in order to support the process of change. The process of language between researcher and researched is made of four interconnected stages describing different levels of knowledge about problems to be faced during the process of change : quasi-ignorance stage, critical theorems stage, enlightenment stage and selection of strategies stage. In the quasi ignorance stage both the researcher and the researched have few concerns about any issue regarding current or potential conflicts between the social and the technical elements. In this stage they begin a discoursive process about the nature of important variables and connection between the two worlds. In the following stage - the critical theorems stage - the researcher begins to explore more about the functioning of the accounting system and the relationship between social and technical elements. The third stage - the enlightenment stage - both researcher and researched develop some common understanding. In this stage the researcher, usually through a kind of action research, identifies all technical and social roots. Then researcher tries to provide deep explanations that should be useful in driving the change. The last stage - the selection of strategies - deals with the individuation of how the change should occur. Strategies could be three: a change in the social aspects, a change in the technical elements or a mutual adjustment between the two elements.

We are interested in studying the process of change of elements within healthcare organization, process of change that has been triggered by a strong external disturbance coming by the regional level. As described in the following session, the process of change is still in course: the external disturbance has created a sort of confusion in organizations that face difficulties in finding a new equilibrium between internal elements (management accounting system, organizational structure and culture).

\section{Changes occurred in Healthcare Sector, of Tuscany Region and the contextualization of the theoretical model}

The Italian national health system (Servizio Sanitario Nazionale: SSN) traces its origins in the "Institution of the National Health Care Service Act" of 1978 (Marcon and Panozzo, 1998; Jacobs et al., 2004). Reforms faced by the Italian Healthcare Sector have seen a progressive decentralization of power to the regional and to the local level (Local Health Authorities -LHAs), the promotion of a policy of diffused services, the introduction of patients' freedom of choice of their healthcare (HC) providers with a creation of a sort of internal market competition and a distinction between hospital care and outpatient care (Marconi, 1997). Also a perspective method of payment was adopted: the DRGs (Diagnosis Related Groups). It was based on a fixed perspective payment and shifted substantial cost risk to hospitals (Evans et al., 1997) creating the need for more sophisticated approaches to hospital budgeting and costing (Kerschner and Rooney, 1987). 
Moreover, while previously the emphasis was on political compliance, reforms of the nineties put emphasis on performance and results. A fundamental distinction of responsibility was highlighted: politicians are responsible for policies and goals setting while managers are responsible for the administration and accountable for achieving results. Under this scheme salary of managers should vary according to performance (Marconi, 1997).

The objective of these reforms was to improve the efficiency and the accountability of local and regional governments, as well as healthcare, school and university by subordinating the public sector to the private sector operational models in order to guarantee greater efficiency of services providers (Kurunmaki, 1999).

The actual Italian HC System is organized in twenty-one regions (and therefore $21 \mathrm{HC}$ regional systems), while at the local level there are 228 Local Health Authorities (Aziende Sanitarie Locali, $L H A s$ ) with a relatively high level of decentralization from the regional level. The local level is accountable for the efficiency and the effectiveness of the health care delivery (Abernethy and Vagnoni, 2004) even if the State still maintains a predominant role in the provision of health care.

Each LHA runs three kind of services (acute care - Hospital-, primary care - Cure primarie - and public health - Prevenzione) as a unique trust. There are also the teaching hospitals (THs) which are independent.

This study has been developed in one Italian Region, Tuscany, and it has been conducted at the LHA level. In Tuscany region there are 12 LHAs and 4 teaching hospitals.

Within the framework of the aforementioned National reforms, the Tuscan Regional authority has introduced innovations in tools and control procedures, now more oriented to the promotion of efficiency and effectiveness. We can recall: the introduction of a Regional Performance Measurement System, the promotion of primary care, the introduction of a new organizational structure based on intensity/severity of care.

The focus of this research is on the organizational structure changes incurred. They have been introduced about in 2006 with the objective to promote visibility of actions, better organization of work and higher efficiency in healthcare sector.

In particular the Regional Government decided that the traditional vertical structure based on units and department had to be re-organized in a horizontal organization based on clinical pathways.

In vertical organization arrangement hospitals were divided in units and departments. Units corresponded to clinical specialities (i.e. orthopaedics, gynaecology, nose and throat speciality) and were grouped in departments such as medicine and surgery.

Every department and unit had its own manager (a medical doctor). There were three levels of accountability: unit manager, department manager and hospital manager.

- Unit managers were responsible for performance and resource consumption in their units. Recourses assigned were personnel (other medical doctors and nurses), beds in their ward, drugs, prosthesis and consumables. They were assigned to both managerial and clinical goals in terms of resource consumption, efficiency and quality in their units. Unit managers answered for the correct management of personnel (i.e. shift, holiday), but were not accountable for the cost of the personnel because they were not really able to influence them. They could only require additional personnel if they demonstrated a real necessity.

- Department managers were responsible for the resource distribution within their departments, but not for resource consumption in units belonging to the department. They dealt with the integration of the units belonging to their departments. They were assigned to more general goals depending on goals assigned to units. 
- Hospital manager was responsible for the performance of the whole hospital. He/she had to combine the strategy of the hospital with the goals assigned to departments and units and had to decide the right distribution of resources.

This organizational structure was represented by an accounting system based on cost centres. This system produced reports underlying costs and performance indicators for units, departments and for the whole hospitals. Performance indicators were mainly indicators in terms of output (i.e. number of surgeries, number of treatments, number of hospitalizations) and in terms of efficiency

The vertical organizational structure presented three problems. First, the defined level of accountability didn't represent the actual distribution of responsibility and the integration between units/specialities/professional in providing healthcare services. In fact nurses play both managerial and clinical tasks: in fact they have to manage a set of resources (consumable, generic drugs and other nurses) and they provide assistance to patients during their stay in hospital. This role was not clear looking at the previous organizational structure. Consequently nurses were not able to perceive completely the importance of their role and on the other side doctors have never accepted the importance of the role played by nurses. Moreover healthcare services are the result of many activities carried out by many units/specialities/professionals. This organizational structure didn't underline this integration and possible problems in the process of services providing. Moreover doctors, proud of their autonomy, often didn't recognize or see the importance of integration and communication about the health of their patients: for example every doctor knows treatments he/she has administered to his/her patient, but he/she doesn't know treatments administered by other doctors. Secondly, the vertical organization was not able to underline causes of outputs and consequently it was not really able to promote improvements of performance and higher efficiency. Thirdly, it was not able to represent the work flow of professional activities and the complexity of healthcare services and treatments.

The accounting system used to support the vertical organization was based on cost centres and presented similar problems with the further problem of producing distorted information for the improvement of performance. First of all, this system defined accountable for costs and goals achievement individuals who were not completely able to influence them and didn't underline the interaction of individuals, thus making unclear the responsibility for results. Secondly, it underlined only the output (in terms of costs or of activities) and not the causes, thus making difficult the individuation of behaviours needed in order to act on final output. Thirdly, the cost due to the complexity of health services provided was not represented, thus making medical doctors not able to use the system for managerial purposes (Mintzberg, 1983).

It is evident that the vertical organizational structure and the relative accounting system were not able to impact significantly on the improvement of efficiency and of performance.

In a context of limited resources the pressure for a greater efficiency has been growing and the Regional level decided to give LHAs the objective to move toward a horizontal organizational structure based on processes, where the patient is put in the centre of the organization. The criteria chosen by the Regional level for the reconversion of the organizational structure was the intensity of care required by patients and no longer specialities. Four levels of intensity of care have been defined: low intensity, medium intensity, high intensity and very high intensity. Above them there are the medicine and surgery departments, each of them has the four levels of intensity. Within each level of intensity every speciality provides its services, depending on the level of care provided by patient. The structure is like a matrix: the rows indicate level of intensity and the columns the speciality. Within the level of intensity doctors are responsible for patients they threat. There are 
four levels of accountability: the Nurse Manager, the Specialist (ex managers of units), the Head of the Department and the Manager of the Hospital.

The Nurse Manager has a managerial responsibility for his/her level of intensity. There is a Nurse Manager for each level of intensity of care. He/she is accountable for the right management of beds in his/her level, low cost drugs and of consumables. He/she has to manage available beds in order to answer to what clinicians require for their patients.

Every Speciality has its own responsible (the Specialist). The Specialist has a clinical responsibility for clinical results of his/her patients and for the quality of the treatment he/she provides. In terms of resource consumption, the Specialist is accountable for high costs drugs and prosthesis. He/she has to negotiate beds with nurses on the base of their patient's requirements. Both Nurse Manager and Specialists are accountable for the right management of their personnel (i.e., shift, holiday ect.) in order to guarantee services to patients, but are not accountable for the cost of the personnel because they are not really able to influence it. They can only require additional personnel if they demonstrate a real necessity. The Head of the Department deals with the integration of goals within doctors and nurses. The Manager of the Hospital has the same goals of the previous vertical structure.

Figure 1 provides an exemplification of how the organizational structure has changed. 
Figure 1 - Vertical vs. Horizontal Organizational Structure
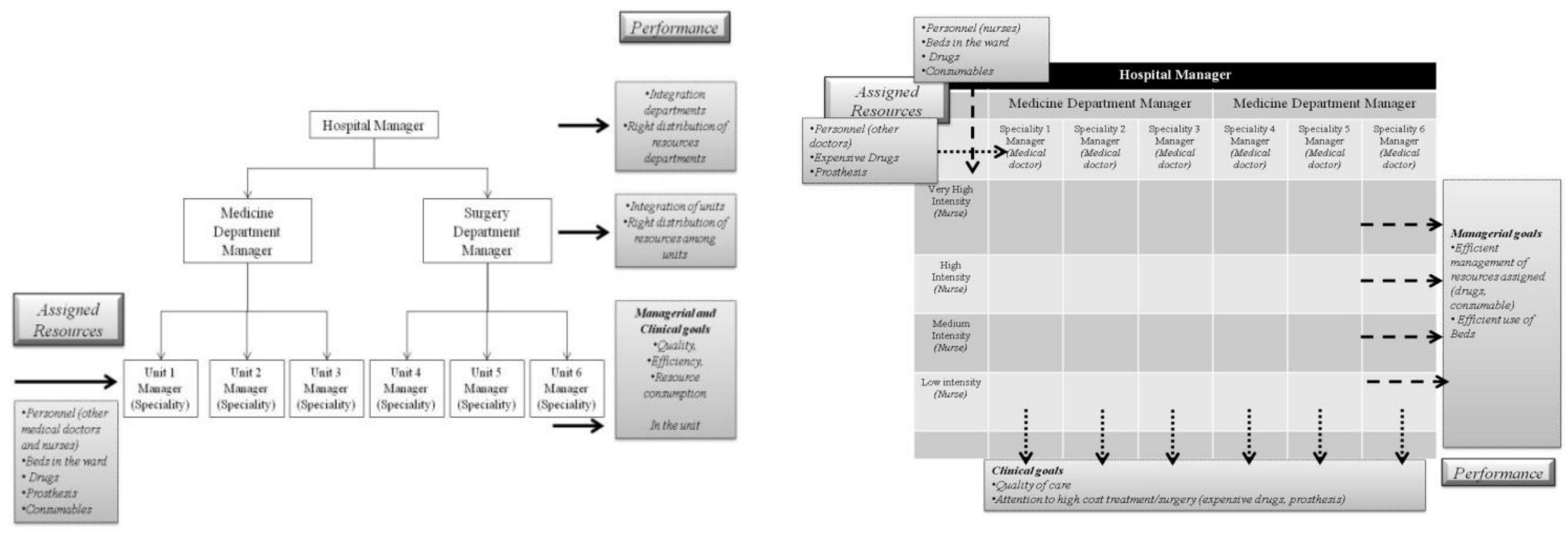

Vertical Organizational Structure

Horizontal Organizational Structure 
The attempt of this organizational structure change is to promote efficiency. In fact it provides the actual representation of responsibility and accountability lines within the organization, put the patient at the centre of the organization and favours a more efficient use of resources.

Regarding the first concern - the actual representation of responsibility - the horizontal organization puts on evidence the role of nurses in the management of beds and consumables in wards. This role, which has ever been played by nurses, in the vertical organization was not recognized by the responsibility accounting, with the consequence that medical doctors were considered accountable for resources that they weren't managing, thus making difficult the improvement of efficiency in their use.

Regarding the second concern - the possibility to put the patient at the centre of the organization the new organizational structure is based on clinical pathway and the minimum unit of analysis is the patient and his/her pathway. This allows underlining also the integration between specialists and professional and their shared contribution in the care of patient, thus favouring the possibility to effectively act on the quality of care.

The third concern - a more efficient use of resources, which is a further consequence of the change in the levels of accountability - also depends on a better management of beds and on an optimization of resources around specific levels of severity of care. In the previous vertical organization each specialist/unit manger had his/her beds and there could be period in which beds and resources were not completely used, thus generating quite high percentage of unused capacity. In the horizontal organization specialists share beds, common drugs and consumables into a certain level of intensity. The sharing of these resources is based on demands for treatments and surgeries. Nurses tend to take up all spaces and to optimize all common resources, with a lower probability of occurrence of unused capacity and supply. Moreover resources are specialized for intensity of care, thus favouring a more correct use of them and avoiding wastes.

In vertical organization variable costs could decrease but fixed costs could remain the same, until a big restructuring or the closing of the ward occurred. It is possible to imagine the reaction of specialists/unit mangers in seeing their ward closed or reduced. On the other side doctors were not really able to influence the demands of treatments or surgeries; they could only act on quality in order to attract patients to their hospital.

The Regional Level, in 2006, started this process of organizational re-engineering. Within general rules provide by the Regional Level, every LHA has started this process of adaptation of the old structure. Every LHA has actuated its own arrangements and modality of application; the process is at an advanced phase, even if many aspects are still to be clarified.

The main problem is the adaptation of management accounting systems, in fact while the organizational structure change has been concluded in many LHAs, the management accounting system still represents the vertical organizational structure. This situation produces incongruence between the work organization and the information provided, making really difficult the decision making process and the evaluation of performance. Moreover the Regional Government had started to ask for information based on the new organizational structure and specific incentives have been established for LHAs that are in a more advanced step of their process of change.

According to the theoretical framework recalled in the previous section (Broadbent and Laughlin, 2005; Habermas, 1987), we considered specific interpretative scheme, sub-systems, design archetypes working in the context of Tuscany HC system and the process of language between researcher and the researched. The specific sub-system we considered is the "Physical organization of space" which means the logistics of the hospital, i.e. where patients are hospitalized, where 
materials are stored etc. The specific archetypes we considered are the "Management accounting system" (MAS), the "Organization of responsibility" and the "Organization of work". The "Organization of responsibility" includes the levels of accountability for performances in the hospital. The "Organization of work" represents the unit of analysis in the organization of work and in the evaluation of results, i.e. the speciality, the patient, the department, the whole hospital etc.

The disturbance has come by the Regional Government and stands in a law which aims to modify the organization of work and responsibility within healthcare organization. This disturbance has provoked a change in the structure of the organization, from a structure at the time t0 (before the disturbance) to the structure at the time t1(after the disturbance)(Figure 2).

Figure 2 - The theoretical framework contextualized

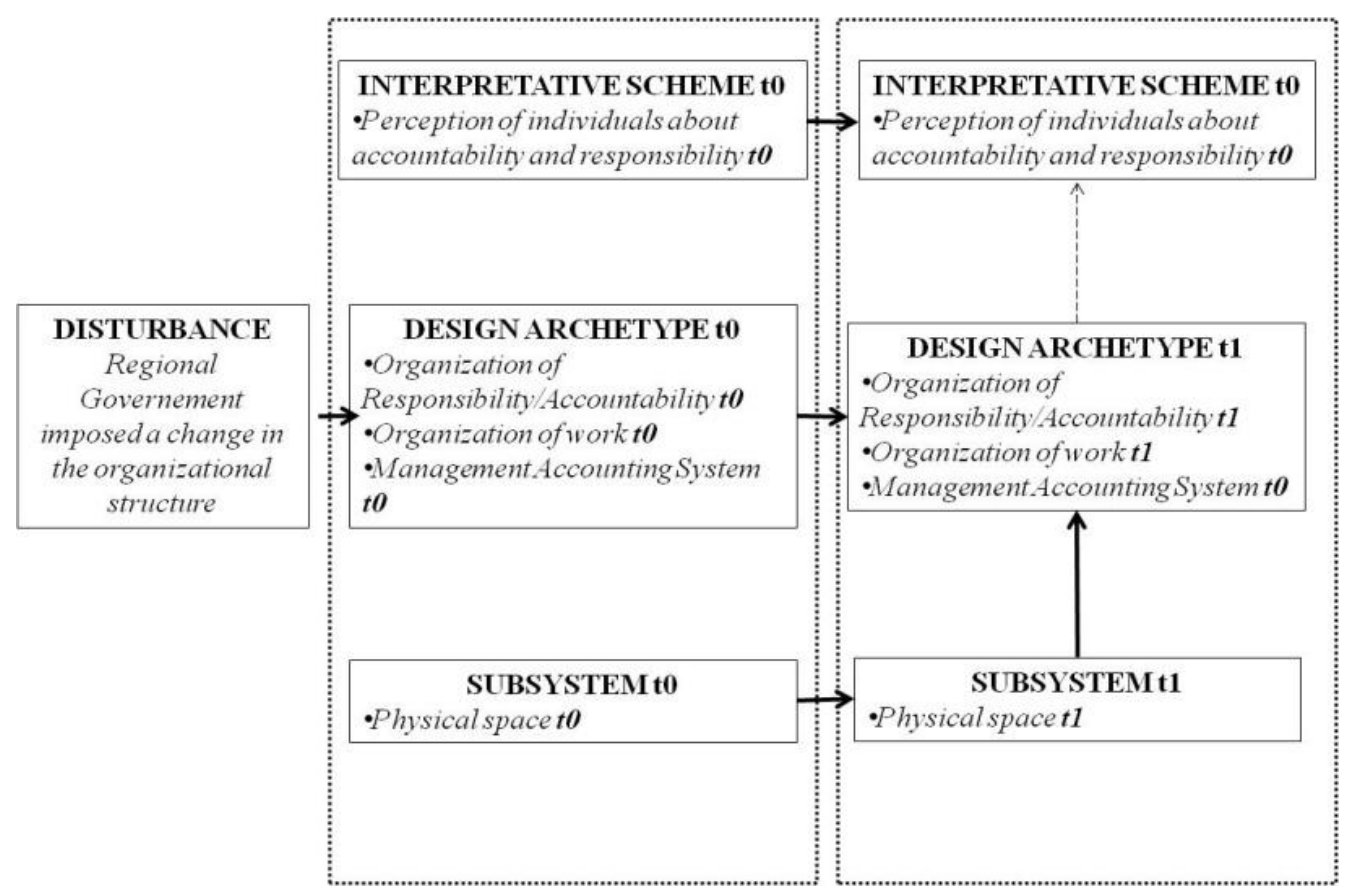

More specifically, today the disturbance has modified two archetypes: the "Organization of responsibility" and the "Organization of work" (but not the Management Accounting System) and the subsystem "Physical organization of space". The Organization of responsibility has shifted from the "Organization of Responsibility t0", with three levels of accountability (Unit Manager, Department Manager and Hospital Manager), to the "Organization or Responsibility t1", with four levels of accountability (Nurse Manager, the Specialist (ex managers of units), the Head of the Department and the Manager of the Hospital). The "Organization of work" has shifted from the "Organization of work" t0, where the unit of analysis is the speciality (orthopaedics, genecology etc), to the "Organization of work" $t 1$, where the unit of analysis is the clinical pathway and the patient.

The subsystem "Physical organization" of space has shifted from the "Physical organization of space t0", with a physical organization of hospital per specialities, to the "Physical organization of space $t 1$ ",with a physical organization of hospital per level of severity of patients treated.

The Management Accounting System has not changed (it is still at point 0)and continue to reflect previous arrangements. 
The interpretative scheme at this moment seems unchanged: more specifically the perception of individuals about the organization of responsibility and accountability is the same as before the external disturbance (it is still at the point t0). The clear evidence is the resistance, showed by clinicians, with respect to the new organization of responsibility. This resistance has been showed by clinicians in many occasions like formal meetings with the regional government and in many meetings and informal talks we have had with them in occasion of education courses or other projects. This resistance is due to a cultural problem. Many controllers told us that clinicians saw these change as a limitation of their responsibility and as a decrease in their power, in favour of an increase in the power and responsibility for nurses. This resistance has also created many tensions within the organizations.

In this situation morphostatis is working: two design archetypes and the subsystem "physical organization of space" have changed with incoherencies and tensions. Also a change in the interpretative scheme is required, because this is the new model imposed by the regional government and because the impact on culture affect the behaviour of individuals, thus affecting performance (Miller, 1981). Moreover the imposed changes represent the actual organization of work and do not answer to political requirements.

The main problem is that a penetration of change in the interpretative scheme requires a change in all the design archetypes mentioned: the management accounting system, the organization of responsibility and the organization of work. In fact clinicians are not able to accept and understand how their responsibility has changed because they can't perceive, in terms of numbers, documents, information etc, the change. Moreover managers, and the regional level are not able to understand performance of hospitals and units.

Considering the two design archetypes already changed, we should underline that, while the new organization of responsibility is completely different from the old one, the organization of work, in the mind of clinicians, has ever been based on patient and clinical pathways; the reform has simply formalized this concept.

With MAS we mean both the budgetary system with its indicators and the cost accounting system with costs of activities, resources etc. These two elements need to change.

Considering the theoretical framework, the following section analyzes the process of change of the design archetype of management accounting system in healthcare sector. This change is made necessary after changes occurred in the design archetypes "organization of responsibility", "organization of work" and in the subsystem "organization of space". In particular we are interested in studying the characteristics of the process of change affecting the possibility of the design archetype MAS, together with the other design archetypes considered, to impact on the interpretative scheme. We are particularly interested in observing the process of change from an internal point of view and in supporting changes using the potentialities of the process of language described by Habermas.

\section{Method, development of the research and outcomes}

Several authors found that a collaborative approach, based on shared meanings, favours the process of change of the interpretative scheme (Dunphy and Doug, 1988; Smith, 1982; Cinquini and Campanale,2010). The design archetype "Management Accounting System" is an important element in providing the alignment between sub-systems and interpretative schemes: in this respect 
the design of the MAS is a critical process. Cinquini and Campanale (2010) found that the equilibrium of the organization can be supported if the design archetype "Management accounting system" is developed within a collaboration and integration between clinicians and accountants. In particular they underlined the importance of the sharing of knowledge among actors.

Considering this, the constructive approach (Kasanen et al., 1993) suits well our purpose. Kasanen et al. (1993) defined the constructive approach "A research procedure for producing construction, where in the management accounting this research approach is intended to produce managerial construction". In their model a new budgeting system or a new method of supporting capital budgeting provide concrete examples of managerial constructions. In their view, accounting is seen as a language and the idea of language fits well with our idea of accounting as a design archetype, that is a process of language used to provide equilibrium within the whole organization.

The constructive approach is characterized by five elements: (1) a problem of practical relevance; (2) a theoretical connection with the problem; (3) the construction of a solution; (4) the demonstration of the practical functioning of the solution; (5) a research contribution to theory. The construction of the solution has to be done within collaboration between users of data, providers of data and researchers. Considering the any complex organization has a tendency to resistance and inertia, the demonstration of the applicability of the solution is the most important factor, together with the innovation to be constructed.

Figure 3 provide an adaptation of the model to our setting.

Figure 3 - Adaptation of Kasanen et al. (1993)

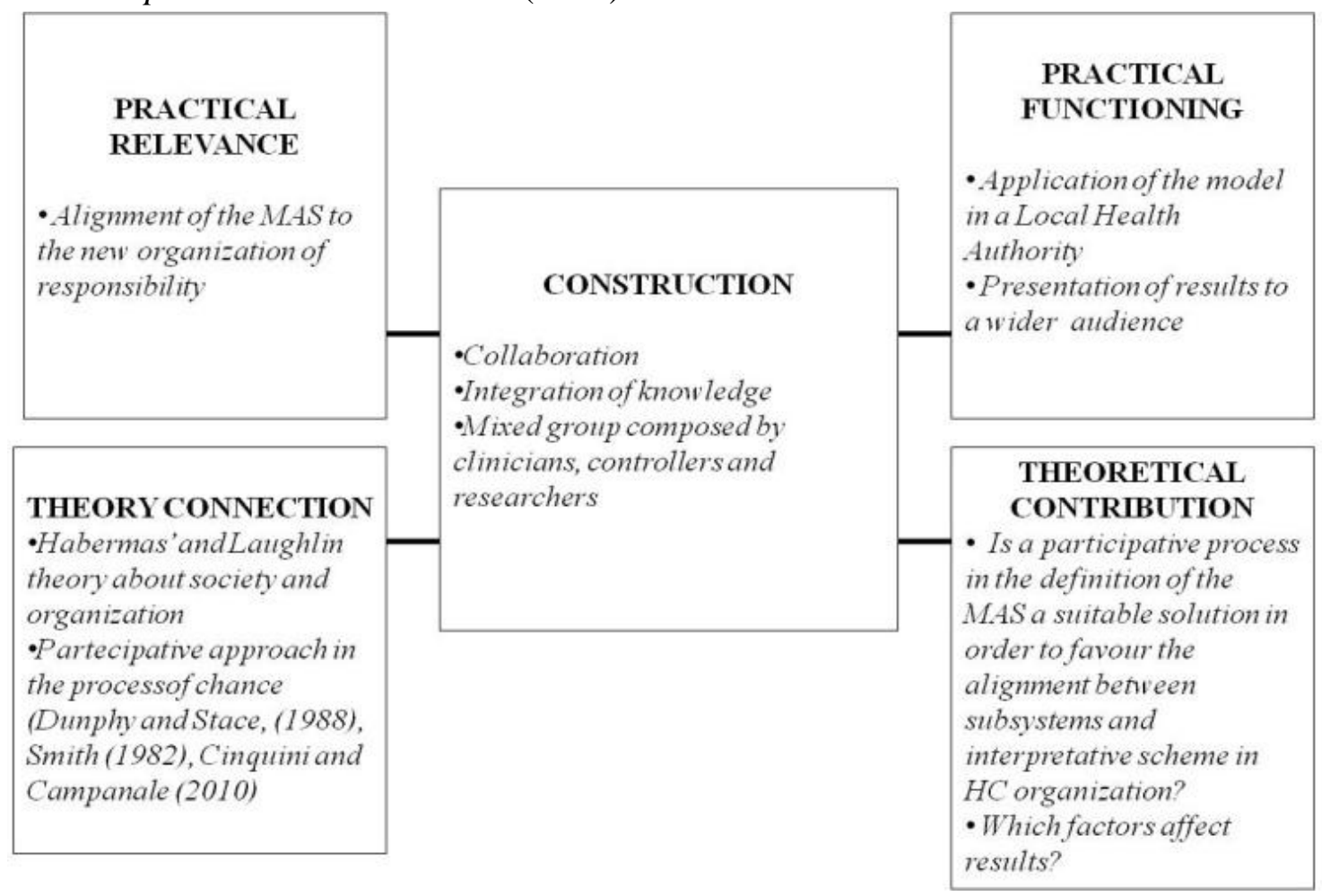

Regarding the application of the constructive approach to our setting, we are able to find all the required elements.

The problem of practical relevance (1), described deeper in the fourth section, stands on the necessity to adapt the design archetype "Management Accounting System" to changes occurred in the design archetypes , "Organization of Responsibility" and "Organization of work" and to 
changes occurred in the subsystem "Organization of physical space". The theoretical connection (2) stands on: (a) the theory of society and organization developed by Habermas and deployed by Laughlin -described in the third section - which underlines the complexity of organizations and the necessity to have an equilibrium between all elements of the organization Particular attention has also to be put in the theory about participative approach in the process of change. In the section we will describe more in deep the other three elements of the approach: (3) the construction of a solution; (4) the demonstration of the practical functioning of the solution; (5) the research contribution to theory.

The construction of the solution (3) has been developed through a collaboration between clinicians and controllers, both belonging to 11 LHAs and $4 \mathrm{THs}$, and researchers. The demonstration of the practical functioning of the solution (4) is an element working on our model, because the theoretical model has been tested and the availability of instruments and information verified.

The contribution to theory (5) will be about factors affecting changes in interpretative scheme and able to determine a morphogenetic change. This element of the constructive approach will be discussed in the final section.

We consider also of particular importance the role of researcher as mediator in the process of change and in the adaptation of elements of the organization. In this respect the steps of the process of language will be described and developed, reminding the four interconnected stages of language (Habermas, 1987): quasi-ignorance stage, critical theorems stage, enlightenment stage and selection of strategies stage. The last three stages have been developed within the steps of the constructive approach. The first stage - the quasi ignorance stage - has been our starting point, because, before the beginning of the research, we already had some concerns about the problem. We learnt these concerns from publications in newspapers, rumours and informal talks.

Element 1 of the approach (individuation of a problem of practical relevance) has dealt with the individuation and the study of the practical problem described in second section. Previously we had some concerns but we needed a shared understanding of the current situation. It comes by interviews with clinicians and controllers of all LHAs and THs and by informal conversations with clinicians. In particular we interviewed 16 controllers and 8 medical doctors. During interviews and informal conversation we shared with participants the views of the problem and started to develop a common language, typical of the critical theorems stage describe by Habermas.

The construction of the solution (element 2) has taken about one year. It has been developed within a mixed group composed by LHAs, THs and researchers. LHAs and THs were represented by both clinicians and controllers. Participation was voluntary. Eleven LHAs and all THs decided to participate, this could be an evidence of the high importance attributed to the problem by organizations. Group meetings were organized about once a month. The group was composed by 35 individuals and coordinated by a controller belonging to a LHA and by researchers. In this step, top managers has not been involved.

In the first five months the group had two main goals: going in dept of the problem and sharing a common language. In first meetings of the group participants shared experiences and problems faced by their organizations and discussed official regional documents. The main objective was to focus on difficulties they found in the adaptation of the MAS to the new organizational structure. Considering implication of the new organizational structure the group planned to focus the attention on two main issues regarding the MAS: (1) how the budget structure should have changed; (2) how the cost accounting system should have changed. 
For both issues the group identified some critical aspects to be faced in the definition of the system: (1) Resources managed by nurses (beds in his/her level, low cost drugs and consumables) are shared by many specialists; (2) the same specialist could act on different levels of intensity of care; (3) how to assign resources to the different levels of intensity; (4) nurses deal both with assistance to patient and with a managerial role; (5) how to map all activities which determine costs and performance. These critical aspects will be described more in dept in the following sessions.

In this step we tried also to trace a set of problems which could cause these difficulties and tried to make some explanations. We identified several factors: (1) a sort of inertia of the organization, (2) a lack of competencies and capabilities of individuals, (3) the availability of dedicated resources, (4) the availability of technologies.

Regarding the inertia - typical of every organization - it seems that these organizations had tried to make some small changes in their MAS, but without investing in technologies and human resources. They made some adjustments to their reports but an effective change in MAS requires to act deep in informative system and an high commitment of people. A deep change requires a group of people dedicated to this task in a full time commitment, while these small adjustments were made by controllers and technicians during their daily work, on a voluntary base. The absence of adequate investments determined all problems linked to the lack of competencies and capabilities (2), and to the lack of resources and technologies (3-4). These experiences however were useful in order to share a common understanding of problems and a common language.

We were not able to act on investments, but we tried to stimulate change and to integrate the lack of technical competencies and capabilities. We tried to reach this goal in several ways: presenting other national or international similar experiences, making proposal, underlying benefits of a possible change. We used a communicative approach with participants in order to gain on trust and create the feeling that we were part of the group. Also our role as members of an external institution, a university which usually works with the regional $\mathrm{HC}$ sector, has been an important factor in determining trust.

In this phase, as researchers, we took notes and started to plan a proposal to be presented to the group, facing the approach of the enlightenment stage of the language described by Habermas.

Next sessions will describe the two relevant issues we faced and how we faced them. Considering the phases of language the identification of solutions traces the phase "Selection of Strategies". The strategy that seems to be chosen is an adaptation of the interpretative scheme. Actually, even if it seems that only the interpretative scheme is adapting to changes in subsystems, the collaborative approach had, in some ways, favoured also the introduction of the culture of individuals in some elements of the MAS developed, described in the following two subsections. This issue will be further developed in the sixth Session.

\subsection{The change in the budget structure}

The discussion about changes in the budget structure took about 1 year and finished with a written document shared by all participants. The most important questions were: "Which are the required behaviours of individuals at the different levels of accountability?", "How can we measure their performance?"; "How the process of negotiation should be?".

Considering these main issues a scheme of document was prepared and then participants started to compile the document. In order to make the process more efficient, usually the coordinator of the group, together with researchers, prepared a proposal to be discussed within the group. 
Firstly the group discussed about problems and opportunities of the new budgetary structure and of the new budgetary negotiation. The budget comprises the assignment of resources and of objectives to be measured with performance indicators.

Table 1 summarize problems and opportunities in building a new Budgetary System.

Table 1 - Opportunities and Problems in the new Budgetary Process

\begin{tabular}{|l|l|}
\hline \multicolumn{1}{|c|}{ OPPORUNITIES } & \multicolumn{1}{c|}{ PROBLEMS } \\
\hline Recognition of the role of nurses & Different role of specialists \\
\hline $\begin{array}{l}\text { Separation between accountability for } \\
\text { management of resources and } \\
\text { accountability for use of resources }\end{array}$ & How evaluate performances \\
\hline More accurate negotiation & More complex negotiation \\
\hline $\begin{array}{l}\text { Possibility to analyze clinical pathways } \\
\text { (pathway indicators) } \\
\text { Higher visibility of actions }\end{array}$ & $\begin{array}{l}\text { Investments in the study and analysis of } \\
\text { clinical pathways }\end{array}$ \\
\hline
\end{tabular}

We remind that in the new organizational structure beds, low cost drugs, nurses and consumables are assigned to the Nurse Manager who manages them. As a consequence, several aspects have to be considered.

Nurse Manager acquires a new managerial role, he/she negotiates beds, low cost drugs, other nurses and consumable with his/her superior. On the other side Specialist negotiates the assignment of personnel (medical doctors), high cost drugs with his/her superior and negotiates his/her use of resources with the Nurse Manager. Both Specialist and Nurse Manager should be involved in the budgetary negotiation with an increasing complexity in the budgetary negotiation. Nurses are accountable for the management of resources assigned to the level of intensity, while Specialists are accountable for the use of those resources. This means that clear roles about the management of resources should be identified and that specific performance indicators, also in terms of efficiency, should be defined at both levels.

Both Specialist and Nurse Manager have to be involved in the budgetary negotiation: the negotiation has to be more accurate in terms of deeper discussions and more documented information for the requirements of resources assignment, but at the same time more complex.

A quite interesting opportunity could be to negotiate resources on the base of patients and clinical pathway. This could favour a higher alignment between activities and necessary resources and could also promote a higher visibility of actions. At the same time investments are needed in order to collect and analyze such more complex information.

These critical aspects were faced by clearly identified actors, behaviours, rules, indicators and time. Together with the coordinator of the group, a table was prepared to be compiled by the group during the meeting. Table 2 summarize such results. 
Table 2 - Level of accountability, required behaviours and performance indicators

\begin{tabular}{|c|c|c|}
\hline $\begin{array}{c}\text { LEVEL OF } \\
\text { ACCOUNTABILITY }\end{array}$ & REQUIRED BEHAVIOURS & PERFORMANCE INDICATORS \\
\hline \multirow[t]{5}{*}{$\begin{array}{l}\text { Head of the } \\
\text { Department }\end{array}$} & $\begin{array}{l}\text { Management of the negotiation in his/her } \\
\text { department }\end{array}$ & \multirow[t]{5}{*}{$\begin{array}{l}\text { All goals assigned to Specialist Manager and } \\
\text { Nurse Manager }\end{array}$} \\
\hline & $\begin{array}{l}\text { Articulation of goals assigned to specialists } \\
\text { and goals assigned to nurses }\end{array}$ & \\
\hline & $\begin{array}{l}\text { Programming of resources necessary for all } \\
\text { department }\end{array}$ & \\
\hline & $\begin{array}{l}\text { Programming of training and education } \\
\text { program for the department }\end{array}$ & \\
\hline & $\begin{array}{l}\text { Management of human resources specifically } \\
\text { dedicated to the department }\end{array}$ & \\
\hline \multirow[t]{4}{*}{ Specialist Manager } & Management of clinical cases & $\begin{array}{l}\text { Number of clinical pathways, medium days of } \\
\text { stay, other goals in terms of quality and } \\
\text { appropriateness }\end{array}$ \\
\hline & Individuation of necessary resources & $\begin{array}{l}\text { Number of needed beds and days of stay, } \\
\text { number of analysis, number of exams, number } \\
\text { hours of surgery room needed etc. }\end{array}$ \\
\hline & $\begin{array}{l}\text { Articulation of objective with other doctors, } \\
\text { also belonging to other specialities }\end{array}$ & $\begin{array}{l}\text { Capacity to reach the goals assigned to the } \\
\text { Speciality through a specific articulation of } \\
\text { goals between doctors belonging to the } \\
\text { speciality. }\end{array}$ \\
\hline & $\begin{array}{l}\text { Take care of the quality of care of all } \\
\text { specialists and doctors in his/her Speciality }\end{array}$ & $\begin{array}{l}\text { Goals in terms of quality, in terms of capacity } \\
\text { to organize adequate training and education } \\
\text { for doctors etc. }\end{array}$ \\
\hline \multirow[t]{5}{*}{ Nurse Manager } & Managing and programming the use of beds & Beds occupancy rate \\
\hline & Managing of other nurses of his/her Level & $\begin{array}{l}\text { Number of hours of extraordinary work, } \\
\text { absenteeism rate etc }\end{array}$ \\
\hline & $\begin{array}{l}\text { Rational and efficient management of } \\
\text { consumable and low cost drugs }\end{array}$ & $\begin{array}{l}\text { Quantity of unnecessary supply, number of out } \\
\text { of date drugs }\end{array}$ \\
\hline & Articulation of goals between nurses & $\begin{array}{l}\text { Capacity to reach the goals assigned to the } \\
\text { Level through a specific articulation of goals } \\
\text { between nurses belonging to the level. }\end{array}$ \\
\hline & $\begin{array}{l}\text { Take care of the quality of care of all nurses in } \\
\text { his/her Level }\end{array}$ & $\begin{array}{l}\text { Goals in terms of quality, in terms of capacity } \\
\text { to organize adequate training and education } \\
\text { for doctors etc. }\end{array}$ \\
\hline
\end{tabular}

Regardless the indicators addressed, what it is interesting is to put attention in the change that seems to be in course.

We simply provided an empty table and the group was able to compile it. They wrote exactly the way behaviours were required to change with the new organizational structure. It seems that some changes in the interpretative scheme are in progress here, because they were able to recognize what the new organizational structure required in terms of levels of accountability.

The group wrote also a document describing how the budget negotiation should be articulated. They identify for each step time, activities and individuals involved. The process of budget they identified was quite articulated and comprised several interactions between Controller, Doctors, Nurses, Head of Department, General Manager of the LHA. For shortness we do not report this document. 


\subsection{The change in the cost accounting system:towards a TDABC approach}

The discussion about changes in the cost accounting system required about 13 months. In order to guarantee the practical functioning of the new cost accounting system, the development of the theoretical model has been contextual to its application in the Teaching Hospital that offered its availability to test the model in its organization (we will call it TO1).

The most important questions were: "How do the new organization of work is represented?", "How do the costs per level of accountability are individuated?", "How the cost information should be organized in order to favour a better informed budget negotiation?".

First of all the group discussed about problems and opportunities of the new budgetary structure and the new budgetary negotiation required.

Table 3 summarizes problems and opportunities in building a new Budgetary System.

Table 3 - Opportunities and Problems in the new Accounting System

\begin{tabular}{|c|c|}
\hline OPPORUNITIES & PROBLEMS \\
\hline $\begin{array}{l}\text { Availability of an organization of cost } \\
\text { information coherent with the new } \\
\text { organizational structure }\end{array}$ & \multirow{3}{*}{$\begin{array}{l}\text { Cultural difficulties in starting to see cost } \\
\text { in multiple perspectives: nurses, } \\
\text { specialists, patients } \\
\text { Necessity of time, capabilities, } \\
\text { investments in order to implement a new } \\
\text { system }\end{array}$} \\
\hline $\begin{array}{l}\text { Possibility to have the cost of activities } \\
\text { performed by both specialists and nurses }\end{array}$ & \\
\hline $\begin{array}{l}\text { Opportunity to have a better resource } \\
\text { assignment }\end{array}$ & \\
\hline $\begin{array}{l}\text { Higher responsibility for all individuals } \\
\text { which could bring to an higher control on } \\
\text { costs }\end{array}$ & $\begin{array}{l}\text { Higher visibility of action which could } \\
\text { bring to resistance }\end{array}$ \\
\hline $\begin{array}{l}\text { Possibility to develop a new system based } \\
\text { mainly on clinicians requirements and } \\
\text { more understandable by them }\end{array}$ & $\begin{array}{l}\text { Necessity of clinicians available to spend } \\
\text { time in the development of the system, } \\
\text { with a partial limitation of time devoted } \\
\text { to the care of patients }\end{array}$ \\
\hline
\end{tabular}

The table underlines the potentiality that a new system could bring to the management of resources within the organization, but also difficulties to be faced. Firstly, there is the opportunity to produce cost information aligned with the new organizational structure and with the actual work flow developed. Discussion of the group suggested the requirement to move from cost per unit to cost per patient. In fact cost per patient is the base for determining cost per unit and per level of intensity. A system based on patient and clinical pathways could favour also a better distribution of resources, in fact, it allows the distribution of resources based on the number and on the complexity of clinical pathways. However, higher investments in terms of training and education, people and technologies are required. Moreover cultural resistance could arise. In fact the new system could generate a shift of power within the organization and there is the risk of a non consonance with the local culture. Considering the nature of professional organizations such as hospitals (Mintzberg, 1983), behavioural and organization variables are key elements that influence the success of innovative technique (Shields and Young, 1989). Some examples are the support provided by the top management, the level of training and resources spent in the project, the clarity of objectives, the involvement in the process of implementation and an eventual link to performance evaluation (Shields, 1995; McGowan and Klammer, 1997). 
Secondly, the promotion of responsibility at all levels, encourages an higher attention on costs, thus bringing to an expected higher efficiency. On the other side the higher visibility of action could create tensions (see Tuomela, 2005), because the visibility of actions limits the possibilities to protect themselves from scrutiny and questioning (Vaivio, 1999).

Thirdly, the possibility to start from a zero base is an incentive and a stimulus to avoid critical aspects of previous system. One of these critical aspects is the inability of a system based on cost centres to represent the work flow of the organization, thus making information unclear for clinicians. Building a new system gives the opportunity to start from clinicians information requirements. In this respect, the first important thing to consider is that the system has to provide a correct diagnosis of the situation (Malmi, 1997) and this implies that people involved in the development of the system must deeply know the operational context and the working environment, otherwise the model risks not to represent the organization reality.

All these critical aspects suggest the necessity of gradualism and involvement in the implementation of a new Accounting System.

Considering the analysed opportunities and problems, a system based on activities has been considered as the most suitable for this case. The most well known costing technique based on activities is Activity-based Costing (ABC). Issues related to ABC in healthcare have been studied for years in relation to questions such as its potential application and results in healthcare organizations.

According to Hilton (2005, p.786) "ABC is a two-stage procedure used to assign overhead costs to products and services produced. In the first stage, significant activities are identified, and overhead costs are assigned to activity cost pools in accordance with the way the resources are consumed by the activities. In the second stage, the overhead costs are allocated from each activity cost pool to each product line in proportion to the amount of the cost driver consumed by the product line".

Activity-based costing is founded on the assumption that resources (personnel, consumable, machineries etc) are required to perform activities and then the activities are used up by the cost objects (a product, a service, a customer, a patient etc).

Activity based costing application requires several steps: (1) definition of the activities; (2) overhead costs are assigned to the activities through "resource drivers" expressing how many resources are consumed in the development of activities. The cost of activities is defined; (3) the activity costs are then assigned to the cost objects through "activity drivers" expressing the frequency and the intensity of their demands for activities.

Among the advantages of an Activity Based Costing system in a health care setting, firstly it makes the patient care process more transparent and activity costs clearer for clinicians and administrative (Lawson, 1994; Udpa, 1996). In general, if activity costs become clearer, the employees' cost awareness improves: in fact these techniques stimulate them to think about the work they do and about the level of efficiency they use (Lawson, 1994; Udpa, 1996). Secondly, ABC is helpful in supporting the budgeting management (King et al., 1994); ABC information helps in (a) assessing resource consumption accurately (also in a forward looking perspective), (b) allocating resources among organizational units, (c) realizing more detailed variance analysis and (4) helping in the production of a profile capacity usage (comparing available vs utilised levels of cost drivers). A third advantage linked to ABC is that it helps in an higher control of costs. For example Kirton and Hazlehurst (1991) claim that Activity Based Costing promotes a better understanding among accountant of how the service works, provides visibility on where and why the cost incurs, highlighting the potential cost of rework, and supports initiative for the quality improvement. They 
argue also that, if cost drivers are well defined, we could express the complexity of the case mix; in fact costs vary on the nature of the patient, on the type of examination, on the mix of the staff and appropriate cost divers can model the effective pattern of resource. A fourth point on Activity-based costing relates its presumed positive effect not only on managerial performance but also on financial performance as discussed in Antikainen et al. (2005). That paper describes a study performed in a Finnish hospital in which simulation of the profitability of an enlargement of a day surgery unit room and department and simulation of how changes in time and personnel influence the costs of activities and operations were performed by an $\mathrm{ABC}$ system. They found also interesting application in the evaluation of unused capacity, in fact they found that every minute of waiting in the operation room costed over 8 Euros/minute (Antikainen et al., 2005).

Despite the advantages many ABC applications failed because the benefits didn't balanced the high costs incurred in implementing and maintaining the system. It has been observed that $\mathrm{ABC}$ application is often limited to specific departments, facilities or business. On this point Gosselin (2007) recalled the basic unsolved issue of the $\mathrm{ABC}$ paradox, which is that "...despite favourable context for the adoption and implementation of $A B C$ and even though $A B C$ exists since almost 20 years, survey have shown that the diffusion process of $A B C$ has not been intense as it may have been expected." (Gosselin, 2007: p.642). Such lack of ABC diffusion is also recalled in Kaplan and Anderson (2007) as a consequence of ABC pitfalls. They recognize that the interviewing and surveying process necessary for cost allocation in the ABC system is time consuming and costly. Furthermore the frequent estimates of the employees for resource allocation were subjective and difficult to validate. These considerations make the $\mathrm{ABC}$ system questionable in its accuracy of cost assignments. A second degree of inaccuracy comes out when it is clear that ABC ignores the potential for unused capacity. The employees' estimates or the activity drivers are considered at $100 \%$ of use of capacity. The authors also noticed that in many companies where $\mathrm{ABC}$ is implemented it remains bounded in individual departments or businesses, not providing an integrated view of enterprisewide profitability opportunities. By the end of Kaplan and Anderson' analysis Activity-based costing appears to be very costly both in implementation and updating and furthermore inaccurate in calculating the cost of the object. The next step in the evolution of $\mathrm{ABC}$ proposed by Kaplan and Anderson (2007) is Time-driven Activity-based Costing (TDABC).

TDABC simplifies the costing process by eliminating the need to interview and survey employees for allocating resources costs to activities; the new system assigns resources costs directly to the cost object in two steps requiring only two estimates. In the first step TDABC calculates the cost of supplying resource capacity (personnel, equipment, technology ect) in a specific department or process. It divides this total cost by the capacity of the department or process (expressed in terms of time) to obtain the capacity cost rate. In the second step the capacity cost rate is used to allocate resource costs to cost object through their demand for resource capacity expressed in terms of time. The two recalled time estimates are: the total capacity of the department or process and the time required to perform an activity.

Considering the opportunities and the problems underlined by the group we proposed the application of TDABC; it is a techniques based on activities but simpler compared to ABC. TDABC seemingly provides many opportunities to design cost models in environments with complex activities, as in healthcare organizations, and service organizations, in general.

During a meeting of the group we presented the rationale of the technique and the recalled advantages in its application in healthcare sector by presenting the paper of Demeere et al. (2009). The paper describes the development and application of a TDABC system for an outpatient clinic in 
Belgium and was very usefull to demonstrate the potentialities of the costing system in such a setting.

We underlined the importance to test the model in an organization in order to link the discussion about the theoretical model to the possibility to see results and understand potentialities. In particular a Teaching Hospital (TO1) demonstrated its availability to test the model. This hospital some years before started a project where all the clinical pathways were mapped, but the project was interrupted after the change of the general director. For this reason, this hospital had a strong motivation in seeing its previous effort re-paid in some ways. This past work permitted to shorten the time of mapping the activities of the pathways and so to concentrate on the other steps of the methodology.

The test started with the application in one department, the department A. The work was organized as follows: within the mixed group, a smallest group was constituted. The smallest group was composed by cost managers and clinicians belonging to the department of experimentation and researchers. This group worked on the requirement of data for the development of the model. Its work consisted in: (1) mapping of processes, (2) identification of actors and other resources involved in the development of activities, (3) identification of time spent by personnel in the development of activities, (4) identification of the cost of resources, (5) collection of all data. Points (1), (2), (3) required the involvement of medical doctors and nurses belonging to the department. In order to obtain the time of each activity three types of resources were defined: medical doctors, nurses and surgery room. The activities and the related times performed by medical doctors were mapped through the interview methodology and they were therefore compared with standards available by professional association. The activities and the times performed by nurses and the usage of the surgery room were recovered by a software.

At every step the smallest (operational) group reported intermediate results to the whole group. Nine meetings with the whole group have been organized. Every meeting was organized as follows: the smallest group reported the work done, underlying straightness and difficulties. The task of the whole group was to discuss the results and to give suggestions in order to continue the development of the model. The operational group, on the base of the suggestions coming by enlarged meeting, modified the model through following steps. These continuous interactions have been useful in order to favour the alignment between the two elements of the constructive approach: the theoretical model and the practical functioning.

AT the end of the explanation of the research steps, we can now summarize in Figure 4 how the process of language described by Habermas in section 3 has been deployed during the process of change. 
Figure 4 - The process of change and the process of language

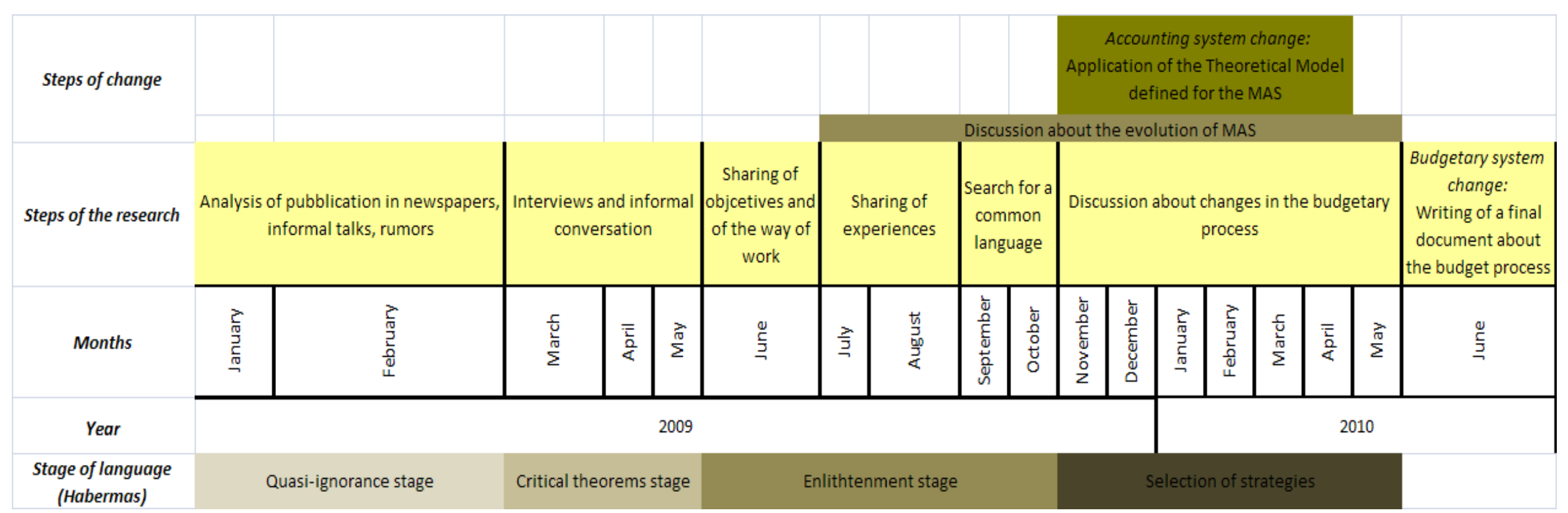




\section{Findings}

The new system and reports provided by this system were presented to clinicians in several occasions, both in non formal and in formal ways: meetings of the mixed group that has worked on the definition of the system, meetings with a larger group of people working on management and control issues, training sessions for clinicians, other informal meetings and talks with clinicians.

Even if we think that changes in the interpretative scheme could be observed in two or three years, first evidences suggest that the approach adopted in the process of change could help clinicians to accept and understand organization archetype and organization sub-system changes and could also bring to cultural changes.

First changes in the interpretative scheme could be observed in many steps of the project: the discussion about the practical problem, the development of the system and the discussion about output.

Clinicians demonstrated a great interest in the discussion of problems linked to the alignment of the management accounting system. Cinquini and Campanale (2010) in a previous research found that, in recent years, the penetration of calculative processes and the attention to cost control, has been affecting deeply the culture of clinicians who started to use cost information to improve performance. This growing culture is in contradiction with the difficult to have information organized on the base of the new organization of responsibility. Clinicians were worried about their inability to have right information for the decision making process. They felt they were losing the control of their performance. In the "Discussion about the practical problem" they were active participants in the definition of problems and relate consequences and gave support in the planning of points to be faced during the project.

In the "Development of the system" phase clinicians gave the highest support: they individuated activities within clinical pathways, personnel and time spent in the development of activities, linkages between activities, pathways and individuals. They also gave useful suggestions in the individuation of how organize information and reports. We, as researchers, only put data into a database in order to elaborate reports.

In the "Discussion about the output" clinicians provided support in the interpretation of results, such as the individuation of the suitable percentage of unused capacity. They also analyze reports in order to compare performance of the units represented, thus providing information about how to interpret differences.

Both clinicians belonging to the group and clinicians who had the opportunity to observe reports were enthusiastic to have information representing their actual work flow, and were happy about the possibility to have deeper information to use in the decision making process, in the evaluation of results and in the budget negotiation.

The possibility to have information about the cost of clinical pathways and about used capacity was particularly appreciated as a tool for the budget negotiation and recourses distribution arrangements. This could allow a higher coherence between activities and necessary resources.

The possibility to have a system able to represent, in terms of numbers, clinical activities could have given an advantage in the possibility of influencing the clinical culture. In fact, as underlined by literature about the professional organizations, systems able to represent complexity of activities are preferred by individuals, particularly by clinicians(, 1989; Jacobs et al., 2004). Moreover also the collaborative approach could have favoured this result, because professionals are more likely to 
accept a system based on standardisation when the system and the supervising derive from their expertise (Abernethy and Stoelwinder, 1990).

\section{Discussion and Conclusions}

This research was developed within the framework of the theory of society and organizations originally developed by Habermas and further developed by Broadbent and Laughlin. Its objective, contextualised in the healthcare sector, was to study the process of change of MAS. Considering previous research (Dunphy and Doug, (1988), Smith (1982), Cinquini and Campanale (2010) we decided to apply a constructive approach (Kasanen et al., 1993) in the development of the new system. We also deployed all phases of language described by Habermas.

In our research context an external disturbance modified the sub-system "Organization of space", thus interrupting the inertia and the equilibrium of the organization. While the archetypes "Organization of Responsibility" and "Organization of Work" changed, MAS had some difficulties, and this created obstacles in the obtainment of an impact in the interpretative scheme, the core of the organization, and thus in generating a morphogenetic, rather than a morphostatic, change. In fact the ability of MAS information to support the representation of responsibility constitute a factor affecting the impact on the interpretative scheme. A morphogenetic change represents a desirable output in our setting, because it could bring to the results expected by regional reforms: higher efficiency, better organization of work and reduction of wastes.

Several elements of the process of change affect this result: (1) the collaborative approach; (2) the role of researchers as promoter of changes, (3) the trust in researchers, (4) the role of researchers as members of a third party legitimated to work in the healthcare sector, (5) good informative systems, (6) the possibility to see tangible results and, according to Grenwood and Hinings (1988), (7) the strength of the contingencies, (8) the level of commitment held by participants, (9) the interests of individuals, (10) the dependences of power, (11) the organizational competences and capabilities oftop managers.

Regarding the first factor, a collaborative approach, it is particularly useful in traditional professional organization where traditional forms of control are usually rejected, according to Dunphy and Doug, (1988), Smith (1982), Cinquini and Campanale (2010). It could favour the adaptation of the new system to professionals' requirements and could avoid the perception of an imposed tool, thus avoiding resistance. Brunson (1985) argued that weak ideologies favour the process of change, while strong ideologies make the change more difficulties. He argue that strong efforts are required in order to overcome problems that could occur in organization with strong ideologies and that a collaborative approach could be useful in overcoming difficulties usually faced in the promotion of changes in organizations with strong ideologies (Brunson, 1985).

Our setting is not characterized by weak ideologies in the sense proposed by Brunson (1985) (where weak ideologies mean inconsistent, simple and inclusive ideologies), on the contrary ideologies are strong. The strengthens of ideologies were evident in the construction of the solution. In fact the group discussed and developed the system considering also its needs and culture. Evidence is the choice to use an Accounting System based on clinical pathways or the choice to develop quality indicators, rather than only efficiency indicators in the Budgetary System. Perhaps the research approach, with the aim to overcome problems traditionally faced in professional organization, has 
favoured a process of change despite strong ideologies. The approach in fact gives the idea of a voluntary and non imposed change.

Regarding the second factor, the role of researchers as promoters of changes, its effectiveness can help organizations to overcome inertia. In fact researchers could make proposals and could help in the application of proposals, thus overcoming partially the lack of competencies, which is a typical factor limiting morphogenetic changes. Also the trust in researchers, the third factor, is an important element. If researchers are perceived as collaborators and not simply as external consultants, there is the possibility to gain high collaboration and thus acquire more information about the problem and be able to propose good solutions. With high trust in researchers there is the possibility that individuals accept and appreciate proposals made by researchers. Trust is favoured also by the membership of researchers to a third party (fourth factor) which traditionally has worked with LHAs and TOs in problem solving.

Regarding the fifth factor, even if the application of TDABC is quite simple, there is the need of instruments and good information in order to process and elaborate data. Moreover the possibility to develop the model and contextually be able to apply it and see some results is a good stimulus to go through next steps of the research and find new results (sixth factor).

The strength of the contingencies (sixth factor) in our setting is evident: the change in the organization of responsibility has created severe contradictions within the organization (between elements of the organization) and between the organization and the external environment. Regarding this last issue, in fact, the Regional Government had started to ask information based on the new organizational structure and specific incentives have been established for organizations able to manage this change, but organizations in that moment were not able to provide information with available MAS.

Regarding the level of commitment of participants, we found low level of commitment with the previous arrangements. This has been evident both during the construction of the solution and both in previous stage of language (the critical theorems stage). During these phases it emerged that individuals where not satisfied with the present arrangement and were willing to a change. Low commitment to the previous arrangement was also evident in efforts put in the process of development of the new system. Regarding dependences of power, we have not empirically data to demonstrate that all groups who have the power were not satisfied with the present system. We could only imagine that top managers and middle managers were not satisfied because they were not able to analyze and improve performance before the solution proposed. If we consider also medical doctor as a power group (as they are the group that in HC settings most of all influence decisions and performances) we can certainly say that they were not satisfied with the previous arrangement and that they were willing to change.

Finally, regarding competencies and capabilities of top managers, we have something different. We are not able to evaluate competencies and capabilities of top managers and their role as promoters of changes because in this phase of the research they have not been involved yet. In fact in the first step of the constructive approach we used in this research, we developed the new Management Accounting System with the contribution of the users of the system. The involvement of the top manager is planned in a second step of the research, in order to implement the new Management Accounting System on a large scale. In this phase a role in the promotion of change has been played by researchers and by the coordinator of the group. Researchers put their skill and knowledge in the process of change. The coordinator was able to promote enthusiasm in the process of change .The 
group didn't have technical competencies but, during the research, researchers had a role in overcome the lack of competencies.

Findings suggest that a morphogenesis is in course. We would observe results in some years but first impressions about the work of the group suggest that a change in the interpretative scheme is in course. Within morphogenetic changes, we should argue that this change is something in the middle between colonization and evolution. Within the phase of language "Selection of strategies" the selected strategy, at first glance, seems a change in the interpretive scheme. However, at the end of the research, we realized that the process of change had resulted in a mutual adjustment between elements of the organizations. In fact the Management Accounting System reflects both the requirement of the Government, in terms of new organization of responsibility, and in part the preexistent culture, in terms of centrality of the clinical pathway, as unit of analysis.

In session 4 we argued that integration of actors and actual distribution of responsibility were not accepted by doctors, proud of their autonomy, but the role of patient as the centre of the organization and the logic of clinical pathways, inside the new organization of work, were something inside the culture of the organization already, even if not formalized. The new interpretative scheme reflects in part something imposed (integration of actors and actual distribution of responsibility) and in part something (the centrality of patient) that was working also before the disturbance. We should argue that this change is something in the middle between colonization and evolution. In fact the disturbance has determined a change in subsystems, design archetypes and consequently in the interpretative scheme (regarding integration of actors and actual distribution of responsibility). At the same time a part of the interpretative scheme (the role of patient and clinical pathways) was already present but has been formalized only after the reform. It influenced the design archetype MAS, which has been defined with emphasis on clinical pathways, activities and clinical indicator.

The constructive approach we used in this research, in some ways, may attempt to make the change in the interpretative scheme less invasive and more aligned with the current culture. The idea of the research was to promote a change in the interpretative scheme by mean of the design archetype Management Accounting System. Findings suggest that, in a situation where individuals are obliged to change their culture, the possibility to use a participative approach, in the development of design archetypes, could favour an higher acceptance of individuals and could make easier the process of change towards a new equilibrium between elements of the organization. The constructive approach used has attempted to make the design archetype Management Accounting System reflecting in part the interpretative scheme and in part the Governmental requirements. The result is that the interpretative scheme can be able, through the Management Accounting System, to continue to influence and regulate the equilibrium between elements of the organization. 


\section{References}

Abernethy M.A., Stoelwinder J.U., (1990), "The relation between organization structure and management control in hospital", Accounting Auditing and Accountability Journal, 3(1), pp. 18-32

Abernethy M.A., Vagnoni E,(2004), "Power, organization design and managerial behavour", Accounting Organization and Society, 29(3-4), pp. 207-224

Antikainen K., Roivainen T., Hyvarinen M., , Toivonen J., Karri T. ,(2005), "Activity based costing process for day surgery unit from cost accounting to comprehnsive management", $B F R$

Broadbent J., Laughlin R. ,(2005), "Organizational and accounting change: theoretical and empirical reflections and thoughts on a future research agenda", Journal of Accounting and Organizational Change ,1, pp. 7-26

Brunsson N. ,(1985), The irrational organization: irrationality as a basis for organizational action and change, London - Wiley

Cassia L., Paleari, S. Redondi R., (2005), "Management Accounting Systems and Organisational Structure", Small Business Economics, 25, pp. 373-391

Chenhall R., Langfield-Smith K. ,(1998), "Performance measure within organizational change program", Management Accounting Research , 9, pp. 361-386

Cinquini L., Campanale C. ,(2010), "Integrative-interactive management accounting in healthcare: evidence from a qualitative research", $33^{\circ}$ EEA Annual Congress

Gosselin, M., (2007), A Review of Activity-Based Costing: Technique, Implementation, and Consequences, in C. S. Chapman, Hopwood A.G., Shields, M.D. (eds) Handbook of Management Accounting Research, Elsevier.

Demeere N., Stouthuysen K, Roodhooft F. ,(2009), "Time driven activity based costing in an outpatient clinic environment: development, relevance and managerial impact", Health Policy, 92(2-3), pp. 296-304

Dumphy D.C., Doug A.S. ,(1988), "Trasformational and coercive strategies for planned organizational change: beyond the O.D. model", Organization Studies ,9(3),

Evans J.H. III, Hwang Y., Nagarajan N.J ,(1997), "Cost reduction and process reengineering in hospitals", Journal of cost management ,11(3), pp. 20-27

Grenwood R., Hinings C.R., Brown J. (1988), "Organizational design types, tracks and the dynamics of strategic change”, $\quad$ Organization Studies, 9(3),pp. 293-316

Habermas J. ,(1987), The theory of comunicative action, Trans T. McCarthy Cambridge Policy Press, Cambridge,

Harris G.G., West A. (1925) ,"A pratical application of scientific methods to hospital accounting", Moderm Hospital, April , pp. 317-318

Hilton R.W., (2005), Managerial Accounting: creating value in a dynamic business environment. New York: McGraw Hill

Jacobs K., Marcon G., Witt D. ,(2004), "Cost and performance information for doctors: an international comparison", Management accounting research ,15(3), pp. 337-354

Jones C.S., Dewing I.P. ,(1997), "The attitude of NHS clinicians and medical managers towards changes in accounting control", Financial accountability \& Management ,13, pp. 261-280

Kaplan R.S., Anderson S.R. ,(2007), "The innovation of time driven activity based costing", Cost Management ,March-April, pp. 5-15 
Kasanen E., Lukka K. , Siitonen A., ,(1993),"The constructive approach in management accounting research", Journal of Management Accounting Research ,5, pp. 243-264

Kerschener M.I., Rooney J.M. ,(1987), "Utilizing cost accounting information for budgeting", Topics in Health Care Finance ,13, pp. 1-9

King M., Lapsley I., Mitchell F., Moyes J. ,(1994), "Costing needs and practices in changing environment: the potential for ABC in the NHS", Financial Accountability \& Management, 10(2), pp. 143-160

Kirton R., Hazlehurst ,(1991), "Activity Based Costing at the Luton \& Dunstable Hospital", CIMA

Kurunmaki L., (1999), "Professional vs financial capital in the field of health care - struggles for the redistribution of power and control", Accounting Organization and Society ,24, pp. 95-124

Laughlin R.C.,(1991), "Enviromental disturbances and organizational transitions and tranformation: some alternative model", Organization Studies ,12(2), pp. 209-232

Lawson, Raef ,(1994), "Activity based costing system for hospital management", CMA Magasine ,68(5), pp. 51-35

Malmi Teemu ,(1997), "Towards explaining activity based costing failure: accounting and control in a decentralized organization", Management Accounting Research ,8, pp. 459-480

Marcon G., Panozzo F. ,(1998), "Reforming the reform: changing roles for accounting and management in the Italian health care sector", The European Accounting Review ,7(2), pp. 185-208

Marconi P. ,(1997), "Public administration reform and government responsivenss to citizens in Italy", Public Management Service - Available at: www.oecd.org

McGowan, A.S., Klammer T.P. ,(1997), "Satisfaction with activity based management implementation", Journal of Management Accounting Research , 19, pp. 217-237

Miller D ,(1981), "Towards a new contingency approach: the search for organizational gestalts", Journal of Manangement Studies ,18(1), pp. 1-26

Miller D., Friesen P ,(1984), Organization: a quantum view, Englewood Cliffs, NJ: Pretice Hal

Mintzberg H ,(1989), The structuring of organizations, NJ: Pretice Hall, Englewood Cliffs,

Mintzberg H. ,(1983), Structure in five: designing effective organizations, Englewood Cliffs, NJ: Prentice Hall

Moores K., Yuen S. ,(2001), "Management accounting systems and organizational configuration: a life cycle perspective", Accounting Organization and Society ,26 (4-5), $\quad$ pp. 351-389

Power M., Laughlin MR ,(1992), Critical theroty and accounting in: Alveson M., Wilmott H. (Eds), Critical Management Sudies, Sage

Robb F.F. ,(1988), Morphostatis and Morphogenesis contexts of partecipative design inquiry in the design of systems of learning and human development, Unprublished discussion paper, University of Edimburgh

Shields, M.D. ,(1995), "An empirical analysis of firms implementation experiences with activity based costing", Journal of Management Accounting Research ,17, pp. 1-28

Shields, M.D., Young S.M ,(1989), "A behavioural model for implementing cost management systems", Journal of Cost Management, Winter, pp. 17-27

Smith K.K. ,(1982), Philosophical problems in thinking about organizational change,in Paul S. Goodman et al (Eds) Change in organization - San Francisco, Jossey Bass

Tuomela T.S. ,(2005), "The interplay of different levers of control: a case study of introducing a new performance measurement system", Management Accounting Research, 16, pp. 293-319 
Udpa ,(1996), "Activity based costing for hospitals", Health Care Management Review , summer, pp. 83-96

Vaivio, J. ,(1999), "Exploring a "non-financial" management accounting change", Management Accounting Research ,10, pp. 409-437

Weick K. ,(1976),"Educational organization as loosely coupled systems", Administrative Science Quarterly, 22(1), pp. 1-19 